\title{
Des langues de spécialité aux documents multimodaux
}

Yves Gambier

\section{(2) OpenEdition}

\section{Journals}

\section{Édition électronique}

URL : http://journals.openedition.org/pratiques/3183

DOI : $10.4000 /$ pratiques.3183

ISSN : 2425-2042

\section{Éditeur}

Centre de recherche sur les médiations (CREM)

\section{Référence électronique}

Yves Gambier, «Des langues de spécialité aux documents multimodaux », Pratiques [En ligne], 171-172 | 2016, mis en ligne le 07 février 2017, consulté le 20 avril 2019. URL : http:// journals.openedition.org/pratiques/3183; DOI : 10.4000/pratiques.3183

Ce document a été généré automatiquement le 20 avril 2019.

(c) Tous droits réservés 


\title{
Des langues de spécialité aux documents multimodaux
}

\author{
Yves Gambier
}

1 Les communications externes et internes d'une entreprise commerciale ou industrielle semblent suivre et entretenir la même logique que son fonctionnement général, à savoir celle, entre autres, de la productivité, de la rentabilité, de l'efficacité.

2 Depuis que l'appellation générique de langues de spécialité (LSP) est apparue à la fin des années 1960, les points de vue des pédagogues sur cette branche de la linguistique (appliquée ?) et les voies d'accès aux LSP se sont multipliés, d'autant plus que les spécialités de domaine sont diverses et en constante évolution et les connaissances spécialisées sont de plus en plus interdisciplinaires.

3 Par ailleurs, les technologies de l'information et de la communication (TIC) ont singulièrement bousculé les manières de concevoir et de distribuer les données, les informations, les messages.

4 Ces trois perspectives - logique des communications d'entreprise, LSP et TIC - exigent de reprendre un certain nombre de concepts et de termes aboutissant aujourd'hui à l'écriture dite professionnelle.

5 Dans un premier temps, on essayera donc de saisir l'émergence de quelques-uns de ces concepts et leur raison d'être. Dans un second temps, on esquissera les évolutions des notions de genre et de texte.

6 L'ensemble cherche à saisir à la fois un passé assez récent et des transformations en cours dans nos façons de lire et d'écrire un texte dit de spécialité. Il s'arrête au seuil des problématiques nouvelles d'écriture experte ou professionnelle pour rappeler les efforts et enjeux des cinquante dernières années qui les ont précédées. 


\section{Des langues dites de spécialité : des appellations ambigües}

\subsection{Un héritage qui perdure}

7 Dès 1977, ont été organisés des symposiums européens biennaux sur les langues de spécialité. Le $20^{\text {ème }}$ a eu lieu à Vienne en Autriche, en juillet 2015. Il portera sur le multilinguisme dans les communications spécialisées à l'ère du numérique. Une analyse des contenus des Actes confirme les tendances lourdes de ces rencontres : elles abordent surtout les aspects didactiques des LSP, c'est-à-dire l'analyse des besoins, les lignes de force des cursus, les manières d'enseigner, les tests et les modalités d'évaluation, approche contrastive, etc. ; elles se penchent surtout sur l'anglais, langue seconde (ESP), via l'étude de traits linguistiques, terminologiques de ces LSP, les problèmes de lecture et de compréhension; elles n'oublient pas pour autant les réflexions méthodologiques (Connor \& Upton, 2004), le rapport à la gestion et à la représentation des connaissances, aux politiques linguistiques, à l'anglais-lingua franca, ni les domaines supposés problématiques (droit, business, médecine, ingénierie, etc.). On peut s'étonner de la permanence des thèmes, de la récurrence des orientations constitutives des programmes.

$8 \quad$ Langue de spécialité est peut-être un hyperonyme excessif dans la mesure où il ne s'agit pas d'une langue à part (même si son lien à la langue dite générale reste ambigu) mais d'une terminologie et d'une syntaxe spécifiques, assorties d'une organisation discursive qui vise l'objectivité de la communication dans une discipline donnée. Ce n'est que progressivement que sont ajoutés des critères pragmatiques de définition : les langues de spécialité sont utilisées et reçues dans des situations particulières - elles s'articulent à certains types d'actes de langage et à des terminologies qui renvoient à des réalités extralinguistiques partagées : objets, pratiques, processus, concepts. En d'autres mots, les LSP tendent à avoir une définition autoréférentielle et tautologique : LSP 㯺 terminologie 䜿 LSP.

9 L'ALAPP (Applied Linguistics and Professional Practices) ${ }^{1}$ qui a organisé quatre conférences internationales depuis 2011 (la cinquième ayant lieu en novembre 2015 à Milan) ne sort pas de la définition circulaire et des thèmes susmentionnés - ciblant toujours l'éducation professionnelle ou plutôt professionnalisante des études en langues, même si son attention porte aussi par exemple sur les dimensions identitaires de ces langues, les demandes institutionnelles. Sa revue (Journal of ALAPP) tente de s'ouvrir à la fois à d'autres pistes d'analyse (multimodale, discursive, communicationnelle) et à des domaines variés d'application.

10 L'Asociación Europea de Languas para Fines Específicos (AELFE), lancée en 1992 (sans doute pour contrebalancer l'anglicisation des Symposiums européens) et dont le $14^{4}$ ème congrès a eu lieu en juin 2015 à Bucarest (Roumanie), poursuit les objectifs didactiques déjà indiqués ci-dessus.

11 Ainsi donc, les LSP demeurent un objet terminologisé, utilitaire (essentiellement pédagogique) et indécis ${ }^{2}$ (Gambier, 1998). Cette indécision, encore perceptible dans le récent numéro de la Revue française de linguistique appliquée (juin 2014), est toute l'ambigüité de «langues" circonscrites tantôt par leur public (d'apprenants ou de spécialistes de domaine), tantôt par leur contenu, les connaissances de référence qu'elles 
sont supposées véhiculer, selon une vision instrumentale de la langue. Elles reflètent ainsi certains découpages (des savoirs, des activités) qui ne relèvent pas des sciences du langage. Prises au sens étroit de technolectes ou de jargons professionnels, elles sont également une manière de reconnaitre l'importance des cultures et des communications technico-scientifiques.

On notera que cette très lente évolution des LSP correspond de facto aux approches audiovisuelles puis communicationnelles dans l'enseignement des langues étrangères, développées dans les décennies 1960-1990. Rappelons que l'approche communicationnelle a mis l'accent, en classe, sur divers types de documents " authentiques ", sur les tâches de savoir informer et savoir s'informer. Les LSP ne semblent pas avoir pris, autour des années 2000, le tournant de la perspective actionnelle, portant sur des projets et visant à faire agir l'apprenant.

\subsection{Multiples étiquettes en français}

13 L'héritage européen, avec ses ambiguïtés, a des traces en didactique du français. On peut ainsi dire que les dénominations de l'enseignement du français de spécialité abondent également, depuis par exemple un manuel de 1927 sur « l'enseignement du français aux militaires indigènes ». On trouve en effet français instrumental, français fonctionnel, français de la communication professionnelle, français de spécialité (plutôt rarement abordé dans les symposiums européens, mentionnés ci-dessus), français sur objectif spécifique (FOS), français à visée professionnelle, français langue professionnelle (FLP), français sur objectif universitaire (FOU), etc. Ces appellations sont à la fois floues, sans rigueur épistémologique et terminologique, tout en se voulant distinctes.

14 Ainsi le français instrumental, dominant dans les années 1970 dans le cadre des méthodologies active et audiovisuelle, devait permettre un accès rapide à la compréhension globale de textes scientifiques et techniques: les apprenants étaient appelés à expliquer, paraphraser, analyser, interpréter, comparer, extrapoler, transposer. On en était alors aux balbutiements de la mobilité des doctorants.

Le français fonctionnel, terme utilisé par le Ministère des Affaires étrangères de Paris à partir de 1974, voulait opérer une rupture avec l'approche lexicale et grammaticale de l'enseignement de la langue : il privilégiait les formations sur mesure, centrées sur les besoins des apprenants.

Le français de spécialité a correspondu (et correspond encore ?) a un enseignement du FLE calqué sur des domaines de connaissances et/ou d'activités, d'où par exemple des manuels du français des affaires, du français médical, du français du tourisme, du français de l'hôtellerie, du français de la banque, etc. Il a donné lieu aussi à des modules de formation en kit et à divers outils pédagogiques, emboitant le pas de l'approche communicative dans l'enseignement des langues. Le français LSP visait des publics étrangers qui ne consistaient pas forcément en étudiants en langue; il demeurait aussi dans des cursus formels, institutionnels, de plus ou moins longue durée, au contraire des FOS (sigle calqué de l'anglais English for Special Purposes). En effet, les FOS correspondaient à des formations sur mesure, brèves, intenses, à objectifs ponctuels, pour des apprenants déjà professionnalisés (Binon \& Thyrion, 2007 ; Mangiante \& Parpette, 2004) ${ }^{3}$. Les FOS ont adopté la perspective actionnelle préconisée par le CECRL (Cadre Européen Commun de Références pour les Langues). 
17 Le français à visée professionnelle, dans la continuité des FOS ou même parfois en parallèle, a reposé sur la maitrise de prérequis et la réalisation de tâches précises, comme savoir se présenter, formuler son $\mathrm{CV}$, passer un entretien, répondre au téléphone, etc. - tout en donnant lieu à des travaux très ciblés, comme par exemple ceux de Damette (2007) sur le français juridique.

Le français langue professionnelle (FLP) s'inscrit davantage dans le cadre des formations professionnelles continues en entreprise (Ferrari \& Mourlhon-Dallies, 2005 ; MourlhonDallies 2007, 2008). Il concerne aussi bien des publics de français langue étrangère (FLE) ou langue seconde que des natifs (français, langue maternelle), loin d'être tous des professionnels avérés, avec une longue pratique de leur métier - par exemple infirmières espagnoles récemment diplômées en partance pour travailler dans des hôpitaux en France. Les objectifs de FLP sont plus larges et englobants que ceux du FOS qui permet à des non-natifs de maitriser le français dans leur contexte professionnel d'origine, en ciblant des compétences limitées (besoins de lecture technique, contacts avec une clientèle française, négociations en français). Le FLP sert souvent des jeunes adultes motivés qui cherchent à s'intégrer dans un cadre de travail en français: la langue est alors perçue comme une des compétences professionnelles.

A cette liste on ajoutera le FOU ou FOA (français sur objectif académique, autre sigle calqué de l'anglais) - sorte de réponse didactique à la mobilité étudiante et à l'internationalisation des études: confrontés aux discours magistraux, aux exigences des essais, des exposés et autres productions écrites et orales propres au milieu universitaire, sinon à certaines disciplines, des didacticiens ont cru nécessaire d'adapter démarches et propositions didactiques du FOS (Mangiante \& Parpette, 2011; Delcambre \& LahanierReuter, 2012). Ils ont alors pris en compte l'analyse des demandes, des besoins, sans négliger l'hétérogénéité des groupes visés, l'impact des nouveaux supports et dispositifs d'apprentissage comme la présentation par diapositives (Powerpoint), le tableau interactif.

On admettra, après ce bref tour d'horizon des années 1970 jusqu'à aujourd'hui, que, selon les appellations, diffèrent :

- les publics ciblés (étudiants en langues, faux débutants en français, étudiants de disciplines non linguistiques, jeunes adultes diplômés à la recherche d'un travail à l'étranger, salariés expatriés, migrants, etc.) ;

- les situations de demande (plus ou moins motivantes) et les contextes (exolingues ou endolingues) dans lesquels prennent place les formations (université, centre de langues, cours de FLE, formation continue, etc.) - supposés favoriser l'autonomisation et la responsabilisation (apprendre à apprendre) des apprenants ;

- les domaines de références (avec implicitement souvent des niveaux d'activité, certains types de relations professionnelles, un statut socio-professionnel: ingénieurs, cadres moyens, employés de secrétariat, personnel plus ou moins qualifié au chômage, juristes, etc.) ;

- les objectifs (liés eux-mêmes au niveau de départ en français, niveau ressenti ou évalué par des tests) et les durées d'apprentissage (cours intensifs ou extensifs).

21 Malgré leur valse, les étiquettes théoriques ont aussi des points de convergence : les méthodes ne sont pas toujours exclusives d'une approche; les stratégies de progression d'apprentissage ne sont pas toujours cohérentes et explicites; les dimensions culturelles des communications sont souvent méconnues ou sous-estimées - instrumentalisant alors la langue et recourant à des contenus stéréotypés, comme si les communications étaient 
vidées d'enjeux, de leur dynamique interactionnelle, c'est-à-dire prenaient place hors hiérarchie, hors de rapport de pouvoir, hors de tout rite de réception...Ainsi un employé de banque parle à des clients, une infirmière s'entretient avec des patients, un juriste fait face à des prévenus, etc. D'évidence, FOS, FLP, FOU...ne sont qu'une des étapes ou qu'une des palettes dans la maitrise du français qui n'est jamais épuré de toute spécificité (même sous son appellation de français dit général, standard, commun). Les étiquettes en question qui visent à promouvoir l'enseignement du français laissent croire qu'on peut compartimenter une langue comme ensemble de termes, de formules phraséologiques, comme moyen d'acquisition et de transformation des savoirs et savoir-faire, comme moyen d'action, comme moyen de reconnaissance et de connivence.

Toute définition et enseignement du français fonctionnel, spécialisé, professionnel... présupposent non seulement une vision de la communication, de la catégorisation des savoirs mais aussi des représentations des langues en général, des compétences déjà acquises en français et en d'autres langues. Ils nécessitent également une pédagogie explicite centrée sur l'apprenant, sur l'apprentissage proprement dit et/ou sur la matière, une prise de position sur la place de la contrastivité et sur la proportion d'enseignement à aborder à l'oral et à l'écrit - sans oublier le rôle des supports hors de la classe (autoapprentissage par exemple grâce aux sous-titres des programmes télévisés, cours à distance, accès aux ressources fournies par l'Internet, etc.).

\subsection{Une langue ou un discours?}

Dans la plupart des travaux sur les LSP, on a le sentiment que la langue sert une communication plutôt linéaire, unidirectionnelle - élaborée du côté du seul énonciateur. Or les LSP, surtout après les analyses pragmatiques qui en ont été faites, portant par exemple sur les actes de langage, l'énonciation, les modalités, sont une forme d'existence de la langue dans une communauté discursive donnée : c'est l'acte de parole, et non la langue, qui devient spécifique d'une situation et d'interlocuteurs partageant en partie au moins un même bagage cognitif et impliqués dans les mêmes types d'activité. Dans cette perspective, l'homologie entre langue et discipline ou profession ne tient pas. Quant aux situations de communication, avec partenaires coprésents remplissant des rôles précis pendant des occupations délimitées (tâches relevant d'un métier, transmission de savoirs), elles débouchent sur des discours sociaux où l'emploi de la langue peut être stratifié - lié aux divisions du savoir (spécialisation) et du travail (hiérarchisation). Ce qui se passe entre le médecin et le malade, le juge et le témoin, le guide et le touriste, le restaurateur et le client met en jeu, comme toute interaction dite ordinaire en langue dite générale, des normes variables de production et de réception langagières. Les communications dites spécialisées dès lors ne sont pas antinomiques des conversations quotidiennes. Tout énoncé, quels qu'en soient la thématique (météo du jour ou avenir de l'énergie nucléaire) et les interlocuteurs, réfère nécessairement à des conventions rhétoriques, stylistiques établies; il s'appuie sur des mécanismes de l'intertexte (rapport à des dits antérieurs, d'où les controverses, les polémiques, les écoles mais aussi les clins d'œil, les connivences), du cotexte (relations signifiantes, renvois entre les parties d'un énoncé), du prétexte (visée, motivation de l'énoncé et situation énonciatrice), du soustexte (allusions, présupposés, implicite, stéréotypes, représentations sémantico-mentales, association d'idées, connotations) et du péritexte (paratexte, ou micro-énoncés annonçant devançant, entourant l'énoncé). 

vulgarisation technico-scientifique : celle des tenants du discours de vulgarisation comme mimétique du discours savant (d'où son aspect mystificateur puisqu'il donne à voir sans les approcher, les comprendre les processus d'élaboration des savoirs) (voir par ex. Jurdant, 1969 et Roqueplo, 1974) et celle des tenants de la vulgarisation comme paraphrase, «traduction» (voir par ex. Jacobi \& Schiele, 1988). On peut considérer la vulgarisation comme reformulation, englobant à la fois les effets du dialogisme et les diverses formes de la paraphrase - la vulgarisation n'étant qu'un des aspects de la reformulation qui inclut aussi des pratiques comme celles du résumé, du discours rapporté, de la synthèse documentaire, de la contraction de textes, de la restitution de données, réalisées au quotidien par des journalistes, des documentalistes, des chercheurs, des rédacteurs, des analystes, etc. D'autres pratiques existent en littérature, dans les performances scéniques (plagiat, pastiche, parodie, etc.), dans l'apprentissage des langues (exercices et activités métalinguistiques, tests et contrôles de connaissances, concept d'interlangue) (Gambier, 1991). La reformulation en dehors de tout enjeu communicationnel a cependant un pouvoir explicatif limité : comme tout autre discours, la vulgarisation prend sens et forme en contexte.

Les rapports entre savoirs, savoir-faire et organisation sociale souligne l'importance de

- la médiatisation du concept à l'énoncé et leur transformation dans un domaine ou entre domaines; 
- l'évaluation constante entre des besoins et pratiques des spécialistes, en rapport avec leur diffusion par les chercheurs eux-mêmes, les éditeurs, les muséologues, les producteurs de films industriels, de vidéo-clips, etc. pour mieux souligner aussi les limitations du concept de LSP, de rappeler quelques transformations en cours :

- Chacun, et tout, est prétendument connecté partout, d'où la multiplication des services et produits.

- Les rédacteurs techniques, les webmestres jouent un rôle-clé pour indiquer comment les services fonctionnent, comment les produits sont utilisables et fiables. Ils fournissent la documentation utile pour installer, accéder, employer, configurer, modifier, réparer, standardiser tel ou tel produit ou service.

- Le consommateur n'est plus récepteur passif: il peut intervenir dans les processus, interagir, proposer - devenant un "prosumer" (mot-valise anglais formé à partir de producer + consumer).

- La globalisation implique à la fois l'internationalisation des processus et procédures de production et la localisation de la distribution. La communication technique est alors de plus en plus intégrée au cycle de vie des produits et services.

- Divers outils technologiques favorisent la création, l'illustration, l'utilisation, la gestion, la mise à jour, le contrôle, la dissémination des contenus documentaires. D'où de nouvelles capacités et organisations du travail de rédaction, d'offres d'information, soumises aux critères d'utilisabilité, d'accessibilité, de lisibilité (Voir 2.3). Désormais, les rédacteurs ne sont plus uniquement tournés vers les clients; ils collaborent aussi avec d'autres spécialistes de l'organisation.

On s'éloigne ainsi des LSP, « langues » perçues surtout comme objet didactique ou même analysées dans la perspective de la "rhétorique contrastive " (Kaplan, 1966), entendue comme étude des différences structurales entre des compositions issues de contextes culturels variés. 

recoupent souvent des domaines de spécialité. On parle ainsi de traduction juridique, médicale, économique, financière, scientifique, technique, etc., mais avec les technologies de l'information et de la communication, ces appellations laissent place de plus en plus à d'autres, comme localisation, transcréation, adaptation, transediting, médiation langagière, versionisation, co-rédaction. Comme pour la rédaction technique, les conditions de travail, le flux continu des informations changent la donne, au point que spécialisation en traductologie tend à signifier désormais au moins trois orientations différentes. La première réfère à des domaines, comme on vient de le signaler (sens dominant au moment de l'émergence des études en LSP); la seconde réfère à des méthodes ou des outils (comme par exemple la traduction audio-visuelle, la localisation de logiciels, de sites Web, de jeux vidéo, ); enfin, la troisième réfère à des genres de texte (comme par exemple la traduction de brevets, de directives de l'Union Européenne, de papiers de recherche en nanotechnologie, la traduction de textes procéduraux, la traduction institutionnelle).

Aujourd'hui, les pratiques et recherches en traduction connaissent un double changement paradigmatique (Gambier, à paraitre) : celui du passage du paradigme de l'équivalence, basé sur la comparaison des textes source et cible, sur la substitution supposée d'un mot par un autre, au paradigme culturel, c'est-à-dire de la traduction orientée non plus vers le texte de départ mais vers la culture réceptrice. À ce changement relativement récent s'ajoute un deuxième: du paradigme de l'imprimé (qui a servi de base au paradigme de l'équivalence) au paradigme du numérique où le texte devient multimodal et volatil (Voir 2.3).

Ce double et rapide changement paradigmatique a lieu maintenant et explique en grande partie la pluralité des étiquettes mentionnées ci-dessus.

On y ajoutera un défi qui touche aussi bien la rédaction technique que la traduction: l'anglicisation de nombre de communications, de données, d'informations qui circulent désormais - à la fois dans le monde des affaires, des sciences, des innovations technologiques et des formations. L'univers des LSP, mis en rapport avec toutes ces transformations, semble s'être bien figé, sinon replié sur le seul enseignement formel.

\subsection{Le filtre des genres et de l'éditologie} objet de recherche et d'enseignement, que si elle est prise en contexte, saisie comme discours situé, avec des agents sociaux.

Un autre élément semble important : celui du genre, avec ses conventions et normes à la fois de production et de réception. Le concept de genre, initialement restreint aux études de folklore et de littérature, a été élargi en analyse de discours et en LSP. Bhatia (2004) l'a repris pour couvrir les professions judiciaires et commerciales. Les genres sont souvent hybrides, associés à des conditions d'emploi, des occasions sociales - par exemple le code de procédure pénale avec un procès, un bon de commande après une négociation. Ce sont des constructions dynamiques qui changent dans le temps et en fonction des cultures, tout en continuant à être perçues comme genres (Frow, 2005). Des documents sur la sécurité, des manuels d'instruction, des accords de partenariat peuvent être soumis à des règles normatives tandis que des rapports annuels d'entreprise, des brochures de marketing peuvent être sujets à variation. Dans une approche fonctionnelle, les genres ou 
types de texte recoupent souvent les diverses fonctions de Roman Jakobson. Ainsi Werlich (1975) a proposé cinq catégories (narration, description, exposition, argumentation et instruction) ; de Beaugrande et Dressler (1981) ont suggéré sept types (descriptif, narratif, argumentatif, scientifique, didactique, littéraire et poétique); Hatim et Mason (1990: 153-160) ont développé trois types principaux dans le cadre d'une réflexion traductologique (argumentation, exposition et instruction) ; enfin, Adam (1992) a défini cinq types (narration, description, argumentation, explication et dialogue). D'autres taxinomies ont été élaborées, certaines reposant davantage sur des stratégies rhétoriques, des modes d'organisation textuelle que sur les fonctions de communication. Quoi qu'il en soit, un mode d'emploi et un communiqué de presse ne fonctionnent pas à la manière d'un exposé savant démonstratif, d'un rapport de laboratoire. Il est facile à ce stade de voir les limitations des taxinomies: les genres ne peuvent être réduits à des catégories tranchées et stables.

Dans tous les cas, et à des degrés divers, l'auteur se met en scène, parfois pour s'effacer complètement (exemple de la recette). Il construit son objet, notamment en se situant dialogiquement (Gil-Salom \& Soler-Monreal, 2014), en reformulant des propositions déjà émises (quitte à s'y opposer), en montrant ses preuves, en interpelant les lecteurs, en jouant sur les niveaux référentiel, logique, linguistique, énonciatif, métalinguistique, rhétorique, terminologique, scriptovisuel. Le nom des auteurs (énonciateurs et cités) font plus ou moins autorité, les textes auxquels on renvoie étant déjà autorisés. C'est à ce prix que se gagnent légitimité et expertise (Voir Bhatia (2004:130-146) sur les rapports entre expertise et genres textuels).

41 Les dimensions interactionnelle et intertextuelle de la communication trouvent un prolongement dans l'étude des transformations éditoriales d'un texte spécialisé, avant sa version finale (imprimée ou électronique). Les réflexions en éditologie mettent en valeur la cooccurrence de la génération de connaissances nouvelles et de leur transfert parce que l'innovation scientifique et technique est fondamentalement un travail à dimension sociale (étude des acquis, montage d'expériences en fonction de ces acquis et de la demande, recrutement de techniciens, pourparlers avec les fournisseurs de matériaux et d'instruments, recherche de financement, prise de brevets, diffusion des résultats, etc.) ; aux critères d'évaluation par des pairs s'ajoutent des critères de commercialisation soupesés par la maison d'édition. Pour l'éditologie, toute spécialité est un ensemble d'écrits édités, selon certaines procédures. En étudiant les processus par lesquels les savoirs sont mis en circulation (commission de contrôle, stipulations d'un contrat, comité de lecture, jury d'acceptation, normes de rédaction...), on met l'accent sur la transmission de l'information ou des données à la fois comme partie intégrante de la formation du discours de spécialiste et comme partie constitutive de la scientificité, puisque les écrits passent par ces circuits d'évaluation où se conquièrent le statut, l'autorité, la crédibilité de l'expert (Callon, 1989 ; Latour \& Woolgar, 1979). Des papiers de travail, des documents de la « littérature grise » des notes de laboratoire aux publications parues, officielles, le travail discursif est incessant, soumis à des filtres différents (valeurs, autorités, conventions linguistiques, guide de rédaction). De nouveau, puisque la «fabrication » des savoirs ne va pas sans une visée de diffusion, on ne peut pas se contenter de LSP comme jargons, ou "styles» vite réduits à une terminologie. Par ailleurs, la logique de la recherche, contrainte par son objet, diffère de la stratégie de son exposé, contrainte par des conventions (éditoriales, académiques). C'est cette communication entre spécialistes - flux incessant entre analyse textuelle, politique éditoriale et sociologie des 
connaissances - que les apprenants à l'écriture professionnelle devraient appréhender avant ou pendant leur rédaction.

\subsection{Vers une nouvelle conception du texte}

42 Avec les communications électroniques, on est passé du «texte » analogique, comme suite linéaire, statique, contextualisée de phrases, de séquences seulement verbales au «texte » pluri-sémiotique, combinant plusieurs systèmes de signes (langagier : oral et écrit, visuel : avec images fixes ou mobiles et pictogrammes, chromatique, sonore avec parfois parole de synthèse, etc.), à manipulation ou accessibilité tactile (via le clavier), sonore (par reconnaissance vocale), bientôt olfactive. Ces «textes» numériques sont volatils c'est-à-dire de courte durée, vite mis à jour, révisés, adaptés, sans auteur unique. De l'énoncé à lecture continue, lente, concentrée, on en est venu aussi à une lecture segmentée, rapide, sans fin, transversale (par navigation). Nos aptitudes cognitives, critiques, ont tendance dès lors à être dominées par l'habileté informationnelle (recherche de données).

L'hypertexte se créé par différents liens, par sauts successifs au lieu d'un défilement séquentiel ordonné comme quand on tourne les pages. La lecture, autocentrée, devient un parcours où chacun construit à la fois son document et son sens, ses blocs d'éléments significatifs : ce qu'on cherche, le but qu'on veut atteindre détermine la sélection des liens par cliquage, zapping ou surf. Chacun (se) construit un « texte » cohérent, à partir de ses attentes et objectifs. L'internaute accumule, intègre des informations, des fragments textuels, élabore sa propre mémoire mnémotextuelle pour arriver à ses fins. On ne lit pas un tel texte sans référer à une interview sur You Tube, à une lecture publique, à une carte, comme on ne regarde plus un film sur DVD sans ses rushes, ses bonus. Dès lors, il s'agit moins de cerner les propos et intentions d'un auteur que de se centrer sur ses propres besoins et motivations.

Ces changements dans les notions de texte, de lecture ne sont pas sans conséquence dans les apprentissages, dans les milieux de travail avec omniprésence d'écrans à consulter, et en traduction : où commence, où finit le site web à traduire ?

Des techniques d'observation comme l'enregistrement des frappes du clavier (keystroke logging) et des travaux en oculométrie ont permis de mettre en évidence l'influence de l'hypertexte, de sa structuration mobile, sur nos modes de navigation, sur nos manières d'appréhender du sens, comment on scanne, infère, extrait une information. Titres, typographie, logo, images...influencent notre parcours ou trajet de lecture. On ne lit pas par exemple modes d'emploi, vidéo clips, brochures touristiques, sites web, publicités de la même façon. On ne saisit pas un «texte » de la même façon si c'est pour s'informer, pour se faire plaisir ou pour le traduire.

46 La traduction audiovisuelle (TAV) est un bon exemple où reconsidérer certains concepts de la traductologie, pris souvent comme allant de soi alors qu'il y a changement de paradigmes (Voir 2.1). Ces questionnements ne valent pas uniquement pour la TAV car nombre de documents sont désormais multimédias et multimodaux. Ils portent entre autres sur

-- La notion de texte: parle-t-on de même «texte» en traduction littéraire, en TAV, en interprétation de conférence? On peut à ce propos rapprocher ici TAV et traduction multimédias (à savoir la localisation de logiciels, d'aides en ligne, de sites web, de jeux 
vidéo et de produits hors ligne comme les cédéroms). Quatre spécificités communes méritent d'être soulignées: en traductologie, cette question a été souvent sous-estimée car l'auteur a été perçu comme unique. En TAV, elle ne peut être éclipsée puisque plusieurs groupes ou institutions interviennent: le scénariste, le dialoguiste, le producteur, le metteur en scène, les acteurs, l'ingénieur du son, le caméraman, le scénographe, etc. Chacun a sa propre compétence (financière, technique, juridique, esthétique) et une autorité variable dans le processus. D'où la complexité des problèmes de droits d'auteur, par exemple lorsqu'un film est co-produit, ou quand déjà sous-titré il est rediffusé sur une chaine de télévision ou diffusé sur le Net.

52 chercheurs en TAV doivent aussi questionner le concept de sens dont la production n'est pas linéaire et ne repose pas sur un système unique de signes. Il y a interaction entre les intervenants, entre eux et les spectateurs, entre les productions AV elles-mêmes (renvois, clins d'œil, allusions). Les hiérarchisations établies entre original et traduction, entre production et reproduction, entre diffusion et rediffusion sont mises à mal, sachant que la propriété intellectuelle d'un film permet souvent, comme aux États-Unis, des montages variés, pour la télévision, sur les lignes aériennes (versions avionnables), ou encore pour des projections politiquement ou moralement correctes, expurgées de toute expression considérée comme injurieuse. conventions dominantes et écrit des sous-titres, entre parler ordinaire et oral du doublage. Plus largement, on peut s'interroger sur les effets à long terme des écritures avec icones, abréviations et émoticons, telles que pratiquées dans les messages SMS, les chats ou conversations en direct. Aujourd'hui, la ville de Montréal offre trois façons d'accéder à son site Internet (www.ville.montreal.qc.ca $\rightarrow$ cliquez sur «accès simple») dont une est en «ortograf altêrnativ» qui mise sur une correspondance orthographique entre lettres (graphèmes) et sons (phonèmes) et qui vise «les personnes qui ont des incapacités intellectuelles» (sic). Ainsi on peut lire «dan bokou de kartié», «lê list dê z'aktivité é dê sêrvis son fêt pour le sitouayin», «alé vouar», etc.

-- Enfin la réception de produits AV est loin d'être une notion consensuelle. Elle peut être considérée à au moins trois niveaux différents: de perception (réactions physiologiques), de cognition (réponses psychologiques et mentales) et d'attitude (répercussions socioculturelles). C'est dire que des éléments comme l'acceptabilité, la lisibilité, la synchronicité doivent s'élargir vers d'autres comme l'accessibilité, la pertinence des informations à transmettre, à omettre, à ajouter, à clarifier, pour ne pas accroitre l'effort cognitif des spectateurs (Voir 2.1). 


\section{En guise de conclusion}

Notre article ne propose pas d'études de cas ni de pratiques d'écriture professionnelle. Cependant, en optant pour une mise en perspective diachronique, il a cherché à relier doutes déjà anciens et préoccupations toujours actuelles - à savoir que l'ambiguïté des LSP réduite à la rédaction professionnelle visant des documents de genres variés ciblant des publics divers ne peut exclure de s'interroger sur la complexité des communications envisagées :

- la maitrise des structures syntaxiques et terminologiques va de pair avec celle des habitudes culturelles, inhérentes à toute composition écrite (le savoir-dire exige un savoir-faire basé sur les rituels et les stratégies de toute composition).

- L'expertise n'est jamais donnée et demande une réflexion conceptuelle avancée car le rédacteur professionnel à «l'écriture experte» ou «de haut niveau» sait qu'il y a une échelle subtile de récepteurs - marginal, occasionnel, ordinaire, compétent, spécialiste, etc. dont les capacités de lecture et les connaissances partagées varient.

- Le professionnalisme du rédacteur s'acquiert à travers de multiples situations d'éducation, de formation, de stage, de pratiques délibérées, de lectures extensives, sans doute grâce à des paliers, des inter-styles, des écritures intermédiaires, des «traces" qui peuvent se fossiliser à un stade donné. En outre, le corps de tels professionnels de la rédaction court toujours le risque de se croire investi du monopole de l'usage légitime d'un certain état de langue, justifiant en un cercle vicieux leur spécialisation.

- Avec les transformations induites par les TIC, peut-on se limiter aux apprentissages individualisés de la rédaction tandis que de plus en plus la production d'écrits est collective, collaborative (Bouchard \& Mondada, 2005), nécessitant une mise en commun de données, négociation argumentée des éléments constitutifs du texte à venir, et cela en face-à-face (oral) ou par l'intermédiaire d'écrans d'ordinateurs, à distance?

- Très souvent, les recherches sur la rédaction sont basées à la fois sur le monolinguisme et sur la séparation des tâches et compétences (entre écriture et lecture). Et pourtant, nombre de travaux aujourd'hui, de nature psycho-cognitive, soulignent combien par exemple la lisibilité et la lecture des textes pendant le processus de traduction, la révision partagent aussi des intérêts communs avec cette rédaction monolingue (touchant la métacognition, les stratégies, l'expertise, le rôle des pauses) et des méthodes communes (avec la saisie d'écran, l'oculométrie, l'enregistrement de clavier ou de frappes). La rédactologie est-elle le signe d'un nouveau figement épistémologique et socio-académique, sous prétexte de dépasser les frontières disciplinaires, les catégories mentionnées en 1.1 et 1.2, ou le signal d'une ouverture pluridisciplinaire, sans a priori conceptuel et méthodologique?

\section{BIBLIOGRAPHIE}

ADAM, J.-M. (1992). Les textes. Types et prototypes. Paris : Nathan. 
AIT EL HADJ, S. \& BELISLE, C. (éds) (1985). Vulgariser: un défi ou un mythe? La communication entre spécialistes et non-spécialistes. Lyon : Chronique sociale.

BEAUGRANDE, R. de \& DRESSLER, W. (1981). Introduction to Text Linguistics. Londres: Longman. BHATIA, V. (2004). Words of Written Discourse. A Genre-based View. Londres: Continuum. BHATIA, V. \& BREMMER, S. (éds) (2014). The Routledge Handbook of Language and Professional Communication. Londres: Routledge.

BINON, J. \& THYRION, F. (2007). « Le français sur objectifs spécifiques : cadrage et mise en perspective ». Le Langage et l'homme, 42 (1), p. 6-23 (numéro consacré entièrement au FOS). BOUCHARD, R. \& MONDADA, L. (2005) : Les processus de la rédaction collaborative. Paris : L'Harmattan. CALLON, M. (1989). La science et ses réseaux. Genèse et circulation des faits scientifiques. Paris : La Découverte.

CONNOR, U. \& UPTON, T. A. (eds) (2004). Discourse in the professions. Perspectives from Corpus Linguistics . Amsterdam/Philadelphie: John Benjamins.

DAMETTE, E. (2007). Didactique du français juridique. Français langue étrangère à visée professionnelle. Paris : L'Harmattan.

DE FERRARI, M. \& MOURLHON-DALLIES, F. (2005). Développer la formation linguistique au titre de la formation professionnelle continue en entreprise. Étude DPM. En ligne : www.clp.asso.fr.

DELCAMBRE, I. \& LAHANIER-REUTER, D. (coords) (2012). « Littéracies universitaires ». Pratiques, 153-154, juin.

FAYARD, P. (1988). La communication scientifique publique. De la vulgarisation à la médiatisation. Lyon : Chronique sociale.

FROW, J. (2005). Genres. Londres: Routledge.

GAMBIER, Y. (1991). « De la reformulation ». In: Gambier, Y. (ed.), Transfer. Viestin Siirto. Turku: Turun Yliopisto, p. 7-26.

GAMBIER, Y. (1998). « Le français dans les communications spécialisées : bilan mitigé ». In: Gambier, Y. (éd.), Discours professionnels en français. Bern : Peter Lang, p. 35-62.

GAMBIER, Y. (à paraitre). « Rapid and Radical Changes in Translation and Translation Studies ». International Journal of Communication vol. 10. En ligne : http://ijoc.org/index.php/ijoc/article/ view/3824.

GIL-SALOM, L. \& SOLER-MONREAL, C. (eds) (2014). Dialogicity in Written Specialized Genres. Amsterdam/ Philadelphie: John Benjamins.

HATIM, B. \& MASON, I. (1990). Discourse and the Translator. Londres: Longman.

JACOBI, D. \& SCHIELE, B. (éds) (1988). Vulgariser la science. Le procès de l'ignorance. Seyssel : Champ Vallon.

JURDANT B. (1969). «Vulgarisation scientifique et idéologie ». Communications 14, p. 150-161.

KAPLAN, R. (1966). « Cultural thought patterns in intercultural education ». Language Learning 16, p. 1-20.

LATOUR, B. \& WOOLGAR, S. (1979) [1986]. Laboratory Life. The Construction of Scientific Facts. Princeton: Princeton University Press. 
MANGIANTE, J. M. \& PARPETTE, C. (2004). Le français sur objectif spécifique : de l'analyse des besoins à l'élaboration d'un cours. Paris : Hachette.

MANGIANTE, J. M. \& PARPETTE, C. (2011). Le français sur objectif universitaire. Grenoble : Presses universitaires de Grenoble.

MOURLHON-DALLIES, F. (éd.) (2007). « Langue et travail : quand faire, c'est dire ». Français dans le monde : recherches et applications 42 , juillet.

MOURLHON-DALLIES, F. (2008). Enseigner une langue à des fins professionnelles. Paris : Didier.

PEYTARD, J., JACOBI D. \& PÉTROFF, A. (éds) (1984). « Français technique et scientifique : reformulation, enseignement ». Langue française 64 , décembre.

ROQUEPLO, P. (1974). « Le partage du savoir. Paris : Seuil. Seconde édition : 1981, Revue française de linguistique appliquée 19(1), juin.

WERLICH, E. (1975). Typologie der Text. Heidelberg: Quelle \& Meyer.

\section{NOTES}

1. À noter que les symposiums européens en LSP et l'ALAPP n'ont pas de site web particulier, avec archives : chaque événement spécifique a ses pages en ligne.

2. Le nombre et la variété des étiquettes témoignent de cette indécision conceptuelle (langages spécialisés, langues techniques, langues spéciales, sous-langues (accent sur le code), langues professionnelles (accent sur les acteurs), langues fonctionnelles, langues savantes, languages for special purposes (accent sur les référents), etc.). La liste n'est pas exhaustive comme le prouve encore la section 1.2.

3. Voir le site Franc-parler (http://www.francparler-oif.org), le répertoire de 20 pages établi par le Centre International d'Études Pédagogiques (septembre 2014) www.Ciep.fr/sites/default/files/ atoms/files/repertoire-methodes.fos.pdf ainsi que le site www.le-fos.com/biblio.htm (consultés le 22.4.2015).

\section{RÉSUMÉS}

La linguistique appliquée s'est soucié, depuis au moins les années 1970, de traiter ce qu'on appelait alors les langues de spécialité (LSP) - manière de référer aux emplois des langues liés à des savoirs et savoir-faire de domaine d'activité et donc de viser à l'efficacité communicationnelle.

Cependant l'objet LSP est resté vague et diverses appellations ont vite pris le relais, sans nécessairement mieux préciser les enjeux à l'œuvre (enjeux conceptuels, pédagogiques, communicationnels, sociologiques).

L'article se développe en deux temps. Dans un premier temps, on essaie de saisir les multiples étiquettes en anglais et en français, leurs ambitions et leurs ambiguïtés, et pourquoi la notion de langue a fait place peu à peu à celle de discours entre divers acteurs sociaux aux statuts et aux 
connaissances différenciés. Ce qui nous amène à préciser le concept d'expertise et aussi à mesurer l'impact de la vulgarisation.

Dans un second temps, on tente d'appréhender certaines forces qui devaient transformer notre perception des LSP, notamment la rédaction technique, la traduction notamment dite spécialisée, les notions de genre et d'éditologie. Sans oublier les conséquences de la numérisation sur notre conception du texte.

La mise en perspective des 50 dernières années permet de relever les défis posés à diverses disciplines par les LSP pour déboucher sur ce qu'on appelle désormais l'écriture professionnelle.

INDEX

Mots-clés : français langue professionnelle (FLP), genre, langue de spécialité (LSP), rédaction technique, texte, traduction

\section{AUTEUR}

\section{YVES GAMBIER}

Turun Yliopisto/Université de Turku, Finlande

University of the Free State, Bloemfontein, Afrique du Sud 